\title{
Rumen anaerobic fungi
}

\begin{abstract}
Of the main types of microorganisms that inhabit the rumen, fungi remain the least characterized group. They have been found to be critical as primary colonizers and digesters of plant matter in the rumen. While they share common fungal characteristics, such as a chitin-containing cell wall, a multi-stage life cycle and arrays of potent carbohydrate-digesting enzymes, they have evolved to an anaerobic lifestyle through other adaptations, such as the hydrogenosome, an organelle which may have developed from mitochondria. Evidence based on comparative genomics is indicating that a number of these adaptations may have involved horizontal gene transfer with other groups of rumen microorganisms.
\end{abstract}

Keywords: rumen, anaerobic, fungi, hydrogenosome, lignocellulosic biomass
Mini Review

Volume 6 Issue 2 - 2018

\author{
Benoit St-Pierre, Venkata Bandarupalli, \\ Prakash Poudel \\ Department of Animal Science, South Dakota State University, USA
}

Correspondence: Benoit St-Pierre, Department of Animal Science, South Dakota State University, Animal Science Complex, Box 2170, Brookings, South Dakota, 57007, USA, Email Benoit.St-Pierre@sdstate.edu

Received: February 27, 2018| Published: March 05, 2018

\section{Introduction}

Harvested as either prey or livestock, ruminants have played a critical role throughout human history, ${ }^{1}$ and they are expected to remain essential in ensuring food security for a rapidly growing and urbanizing global population. ${ }^{2}$ Like other herbivores, ruminants do not encode in their genome enzymes that are effective in breaking down plant fiber. To convert lignocellulosic biomass into utilizable energy, they rely on the metabolic activities of symbiotic microbial communities that reside in the rumen segment of their four-chambered stomach $^{3}$. Ruminal microbial communities consist of complex and diverse assortments of bacterial, methanogenic, protozoal and fungal species, which, as a group, metabolize plant fiber polymers into short chain fatty acids (SCFAs) that are rapidly absorbed by the rumen epithelium. ${ }^{4}$ These rumen-produced SCFAs, a mixture composed primarily of acetate, propionate and butyrate, contribute up to $80 \%$ of the energy requirements of the ruminant host. ${ }^{5}$

Dating back to the work of Robert Hungate and other pioneers of rumen microbiology, decades of research efforts have provided great insights into this complex microbial ecosystem. ${ }^{6}$ In recent years, however, an increasing number of community-level analyses using DNA sequencing-based techniques have revealed that the complexity of the rumen microbial ecosystem is not only far greater than had been estimated by culturing-dependent techniques, but also that the vast majority of rumen microorganisms remain to be characterized. A factor likely to foster such high microbial diversity include the ruminant diet, which can support a wide array of metabolic specialists with overlapping functions. ${ }^{7,8}$ The difficulties experienced in trying to isolate additional rumen microbial species is the result of a number of factors, such as their anaerobic lifestyle and the intricate networks of mutually beneficial trophic relationships amongst members of the community that are required for them to achieve high levels of efficiency. ${ }^{9}$

Of the different types of microorganisms that inhabit the rumen, fungi represent the most poorly characterized group. Due to their low cellular density and the difficulties in identifying them, ${ }^{10,11}$ rumen fungi were initially thought to have only a minimal involvement in ruminant digestion. However, increasing evidence is supporting their function as primary colonizers and digesters of plant matter in the rumen. Indeed, the presence of plant-based fibrous material in the rumen rapidly attracts zoospores, presumably through chemotaxis, which then proceed to colonize this substrate..$^{12-14}$ Once attached, zoospores develop into mycelia, which then invade and break down plant fiber material. As a result of their activity and growth, rumen fungi are able to penetrate and disrupt plant tissue, which also increases the area of exposed substrate, facilitating access to other plant fiber utilizers such as bacteria and protozoa.

The important contribution of anaerobic fungi in ruminal digestion is also well supported by the observation that the rate at which they can metabolize plant matter is not affected by substrate particle size, while digestion rates for bacteria become reduced as the size of substrate particles increases. Anaerobic fungi are equipped with similar tools as their other relatives, breaking down lignocellulosic material by expressing a range of plant fiber metabolizing enzymes in addition to the invasive growth of their vegetative mycelium. Free as well as multi-enzyme complexes, such as cellulosomes, have been identified in rumen fungal species for which genome sequence is available. ${ }^{15,16}$ However, as would be expected from their anaerobic lifestyle, rumen fungi are devoid of mitochondria, and do not possess cytochromes nor other biochemical components required for oxidative phosphorylation. Intriguingly, they use specific organelles, hydrogenosomes, to ferment monosaccharides released from the breakdown of plant structural polysaccharides into $\mathrm{H}_{2}, \mathrm{CO}_{2}$, formate and acetate as end products. ${ }^{15,17}$ Since hydrogenosomes share common characteristics with mitochondria, they may have evolved from them. ${ }^{18-20}$ Rumen fungi are found in close association with methanogens, a mutually beneficial relationship in which the metabolic activity of the latter maintains $\mathrm{H}_{2}$ at low levels which favors hydrogenase activity in the hydrogenosomes. ${ }^{21}$ While they can also produce lactate and ethanol as waste products, hydrogenosome-driven metabolism is likely more favorable. ${ }^{11}$

While they share common fungal characteristics, such as a chitincontaining cell wall and a multi-stage life cycle, ${ }^{10,22}$ rumen fungi appear to be phylogenetically distinct. Indeed, the genera so far 
identified cluster in a separate clade, which have been assigned to their own family (Neocallimastigaceae), order (Neocallimastigales), class (Neocallimastigomycetes), and phylum (Neocallimastigomycota). ${ }^{23}$ They include three monocentric genera (Neocallimastix, Caecomyces and Piromyces) and three polycentric genera (Anaeromyces, Orpinomyces and Cyllamyces), with two additional proposed genera (Oontomyces and Buwchfawromyces) based on molecular data from other gut environments..$^{24,25}$ Comparative genomic studies have revealed that horizontal gene transfer appears to have played an important role in the adaptation of fungi to gut environments, which have included carbohydrate degrading enzymes as well as certain hydrogenosome enzymes. ${ }^{11}$

Slowly, but surely, interest in anaerobic fungi has continued to gain in momentum, in large part driven by interest in their biotechnological potential. ${ }^{11}$ With continued improvements in culturing, phylogenetic profiling, metagenomics, and gene annotation, more insights are likely yet to come for these fascinating fungi.

\section{Acknowledgements}

None.

\section{Conflict of interest}

None.

\section{References}

1. Hackmann TJ, Spain JN. Invited review: ruminant ecology and evolution: perspectives useful to ruminant livestock research and production. $J$ Dairy Sci. 2010;93(4):1320-1334.

2. Thornton PK. Livestock production: Recent trends, future prospects. Philos Trans R Soc Lond B Biol Sci. 2010;365(1554):2853-2867.

3. Hofmann RR. Evolutionary steps of ecophysiological adaptation and diversification of ruminants: A comparative view of their digestive system. Oecologia. 1989;78(4):443-457.

4. Bergman EN. Energy contributions of volatile fatty acids from the gastrointestinal tract in various species. Physiol Rev. 1990;70(2):567-590.

5. Gäbel G, Sehested J. SCFA transport in the forestomach of ruminants. Comp Biochem Physiol A Physiol. 1997;118(2):367-374.

6. Krause DO, Nagaraja TG, Wright AD, et al. Board-invited review: rumen microbiology: leading the way in microbial ecology. J Anim Sci. 2013;91(1):331-341.

7. Wallace JR. Gut microbiology_-Broad genetic diversity, yet specific metabolic niches. Animal. 2008;2(5):661-668.

8. McSweeney C, Mackie R. Micro-Organisms and ruminant digestion: state of knowledge, trends and future prospects; background study paper no. 61; food and agriculture organization of the United Nation: Rome, Italy; 2012.

9. Johnson DR, Goldschmidt F, Lilja EE, et al. Metabolic specialization and the assembly of microbial communities. ISME J. 2012;6(11):1985-1991.
10. Orpin CG, Joblin KN. "The rumen anaerobic fungi". In: Hobson PN, Stewart CS, editors. The rumen microbial ecosystem. Dordrecht: Springer; 1997:140-195.

11. Edwards JE, Forster RJ, Callaghan TM, et al. PCR and omics based techniques to study the diversity, ecology and biology of anaerobic fungi: insights, challenges and opportunities. Front Microbiol. 2017;8:1657.

12. Orpin CG. Studies on the rumen flagellate Neocallimastix frontalis. Microbiology. 1975;91(2):249-262.

13. Bauchop T. Rumen anaerobic fungi of cattle and sheep. Appl Environ Microbiol. 1979;38(1):148-158.

14. Williams AG, Orpin CG. Glycoside hydrolase enzymes present in the zoospores and vegetative growth stages of the rumen fungi Neocallimastix patriciarum, Piromonas communis, and an unidentified isolate grown on a range of carbohydrates. Can J Microbiol. 1987;33(5): 427-434.

15. Youssef NH, Couger MB, Struchtemeyer CG, et al. The genome of the anaerobic fungus Orpinomyces sp. strain $\mathrm{C} 1 \mathrm{~A}$ reveals the unique evolutionary history of a remarkable plant biomass degrader. Appl Environ Microbiol. 2013;79(15):4620-4634.

16. Gilmore SP, Henske JK, O’Malley MA. Driving biomass breakdown through engineered cellulosomes. Bioengineered. 2015;6(4):204-208.

17. Yarlett N, Orpin CG, Munn EA, et al. Hydrogenosomes in the rumen fungus Neocallimastix patriciarum. Biochem J. 1986;236(3):729-739.

18. Embley TM, Horner DA, Hirt RP. Anaerobic eukaryote evolution: hydrogenosomes as biochemically modified mitochondria? Trends Ecol Evol. 1997;12(11):437-441.

19. van der Giezen M, Slotboom DJ, Horner DS, et al. Conserved properties of hydrogenosomal and mitochondrial ADP/ATP carriers: a common origin for both organelles. EMBO J. 2002;21(4):572-579.

20. Voncken F, Boxma B, Tjaden $J$, et al. Multiple origins of hydrogenosomes: functional and phylogenetic evidence from the ADP/ ATP carrier of the anaerobic chytrid Neocallimastix sp. Mol Microbiol. 2002;44(6):1441-1454.

21. Bauchop T, Mountfort DO. Cellulose fermentation by a rumen anaerobic fungus in both the absence and the presence of rumen methanogens. Appl Environ Microbiol. 1981;42(6):1103-1110.

22. Orpin CG. The occurrence of chitin in the cell walls of the rumen organisms Neocallimastix frontalis, Piromonas communis and Sphaeromonas communis. J Gen Microbiol. 1977;99(1):215-218.

23. Wang X, Liu X, Groenewald JZ. Phylogeny of anaerobic fungi (phylum Neocallimastigomycota), with contributions from yak in China. Antonie Van Leeuwenhoek. 2017;110(1):87-103.

24. Dagar SS, Kumar S, Griffith GW, et al. A new anaerobic fungus (Oontomyces anksri gen. nov., sp. nov.) from the digestive tract of the Indian camel (Camelus dromedarius). Fungal Biol. 2015;119:731-737.

25. Callaghan TM, Podmirseg SM, Hohlweck D, et al. Buwchfawromyces eastonii gen. nov., sp. nov.: a new anaerobic fungus (Neocallimastigomycota) isolated from buffalo faeces. MycoKeys. 2015;9:11-28. 